\title{
Comparison of Mangalica and Hungarian Large White pigs at identical bodyweight: 2. Fatty acid regiodistribution analysis of the triacylglycerols
}

\author{
ANDRÁS SZABÓ ${ }^{1}$, PÉTER HORN ${ }^{1}$, RÓBERT ROMVÁRI' ${ }^{1}$, ZOLTÁN HÁZAS' ${ }^{1}$ and HEDVIG FÉBEL ${ }^{2}$ \\ 'Kaposvár University, Faculty of Animal Science, Laboratory of Animal Product Qualification, Hungary, ${ }^{2}$ Research \\ Institute of Animal Breeding and Genetics, Herceghalom, Hungary
}

\section{Abstract}

At identical bodyweight values (130 kg) Mangalica and Hungarian Large White tissue (liver, kidney, heart and skeletal muscle, adipose tissue, spleen and lung) triacylglycerol (TAG) fatty acid distribution analysis was performed, to describe tissue types and possible genotype-associated differences. Tissue TAG was partially hydrolyzed with hog pancreatic lipase, and the fatty acid profile of 2-monoacylglycerols (2MAG) and TAGs was analyzed by gas-chromatography. All extrahepatic tissues provided marked central (sn-2) saturated fatty acid (SFA) (mostly palmitate) recruitment, while liver 2MAGs were mainly aclylated by unsaturated fatty acids (UFA). Inter-genotype differences were minor: in adipose tissue in Mangalica the total palmitate moiety was found in the 2MAGs, while in the liver docosapentaenoic and docosahexaenoic acids were only found in the Mangalica 2MAGs. In conclusion, the direct analysis results of the 2MAGs in six extrahepatic organs and in the liver provided evidence that the building-up of the characteristic porcine TAG structure is located at a post-hepatic site, being true for most of the organs in the pig body.

Keywords: pig, Mangalica, Hungarian Large White, triglyceride, 2-monoglyceride, fatty acid

\section{Zusammenfassung}

\section{Vergleich zwischen Mangalitza und Ungarischem Large White bei gleichem Lebendgewicht: 2 . Fettsäureanalyse der Triglyceride verschiedener Schlachtkörpergewebe}

Bei einem Schlachtgewicht von $130 \mathrm{~kg}$ wurden bei Mangalitza und Ungarischen Large White Triglycerol-(TAG)-Fettsäuren der Gewebe von Leber, Niere, Milz, Lunge, Rückenfett, Herz- und Skelettmuskel analysiert, um diese zwischen den Gewebearten und Rassen zu vergleichen. Die Hydrolyse der Triglyceride erfolgte mittels Pankreaslipase und die Bestimmung der Fettsäuremuster - sowohl der Triglyceride als auch der 2-Monoglyceride (2MAG) - mittels Gaschromatographie. Alle nichthepatischen Organe zeigten eine bedeutende zentrale ( $s n-2)$ Verteilung der gesättigten Fettsäuren (SFA) (besonders Palmitinsäure) während die hepatischen 2MAGs meist mit ungesättigten Fettsäuren (UFA) acyliert waren. Die Differenz zwischen den Genotypen war gering. Im Rückenfett der Mangalitza-Tiere fand sich nur halb soviel Palmitinsäure während Dokosapenaensäure 
und Dokosahexaensäure sich ausschließlich in der Leber von Mangalica fanden. Die direkte Gewebeanalyse der 2MAG in der Leber und sechs weiteren nichthepatischen Organen zeigt, dass das für Schweine typische TAG-Fettsäuremuster in einer posthepatischen Lokation synthetisiert wird und für die meisten inneren Organe charakteristisch ist.

Schlüsselwörter: Schwein, Mangalitza, Ungarisches Large White, Triglyceride, Fettsäuren, 2-Monoglyceride

\section{Introduction}

The positional distribution of fatty acids in animal triacylglycerols (TAGs) is non-random (LEIBETSEDER 1996). HILDITCH et al. (1935) presented results on the special TAG structure of the domestic pig, namely the predominant palmitic acid recruitment into the $s n-2$ position. Further homeotherm examples for this are among others the wild boar and the peccary (MATTSON et al. 1964) and human and porcine milk fat (INNIS et al. 1995). Since colipase-dependent pancreatic lipase is strictly sn-1,3 specific (INNIS et al. 1995), there must exist at least one further pathway in the TAG synthesis of these species over the reacylation of $2 \mathrm{MAGs}$ in the gut endothelium, if isomerisation (via the activity of mutase) is excluded. BROCKERHOFF and ACKMAN (1967) presented data for a pig raised on a fat free diet, also providing the basic preponderance of palmitate at position $s n-2$ in the TAGs. STOKES and TOVE (1975) described a "factor" controlling the regiospecificity of acyltransferases in the porcine TAG synthesis. This "specifier factor « has been localized in the adipose tissue microsomal fraction and was altering the specificity of acyltransferases to synthesize 2-palmitoyl TAGs. Interestingly, the activity of the specifier factor has been established besides adipose tissue in the kidney and heart, but not in liver.

LEE et al. (2001) described a novel, alternative pathway for TAG synthesis, the direct acylation of glycerol, in pig. Glycerol acyltransferase activity was detected in heart, skeletal muscle, liver, kidney and brain microsomal fraction. While glycerol acyltransferase activity is present in numerous tissues, its fatty acid preference concerns palmitoyl- and arachidonyl-CoA, but is not exclusive. However, its role in shaping the special TAG structure in pigs is not clear.

The purpose of the present study was not only to describe the TAG fatty acid structure in numerous, partly not yet analyzed tissues, but to obtain novel data on the autochthonous Hungarian breed, Mangalica. Latter breed has not been investigated in such detail in the past, but is supposed to produce fat of special quality.

\section{Material and methods}

\section{Samples}

Subcutaneous fat, liver, kidney, skeletal muscle ( $m$. longissimus dorsi), heart muscle, lung and spleen were sampled and stored at $-70^{\circ} \mathrm{C}$ until analysis. Two genotypes, Mangalica and Hungarian Large White were involved in the study ( $n=6$ in each case), at identical slaughter bodyweight (132 \pm 9 vs. $130 \pm 8$, Mangalica vs. Hungarian Large White). 
The proximate composition of the two fattening diets was basically similar, as assessed from the packages, and as shown in Table 1. Since Mangalica keeping was extensive, being the characteristic keeping mode for this breed, the composition of the basic and additional unknown feedstuff was analyzed based on its fatty acid composition. For this purpose at slaughter in both genotypes stomach content was sampled, extracted (FOLCH et al. 1957) and analysed with gas-liquid chromatography.

Table 1

Proximate and fatty acid composition of the diets

Futterzusammensetzung

\begin{tabular}{|c|c|c|c|c|}
\hline & \multicolumn{2}{|c|}{ Mangalica } & \multicolumn{2}{|c|}{ Hungarian Large White } \\
\hline Dry matter, \% & \multicolumn{2}{|c|}{88.4} & \multicolumn{2}{|c|}{88.0} \\
\hline Metabolizable energy, MJ/kg & \multicolumn{2}{|c|}{13.5} & \multicolumn{2}{|c|}{13.7} \\
\hline Digestible energy, MJ/kg & \multicolumn{2}{|c|}{13.6} & \multicolumn{2}{|c|}{13.9} \\
\hline Crude protein, $\%$ & \multicolumn{2}{|c|}{11.8} & \multicolumn{2}{|c|}{12.0} \\
\hline Crude fat, $\%$ & \multicolumn{2}{|c|}{4.2} & \multicolumn{2}{|c|}{4.0} \\
\hline Crude fiber, \% & \multicolumn{2}{|c|}{5.0} & \multicolumn{2}{|c|}{5.0} \\
\hline Fatty acid & TAG & $2 M A G$ & TAG & $2 M A G$ \\
\hline $\mathrm{C} 10: 0$ & 0.02 & 0.15 & 0.02 & 0.11 \\
\hline $\mathrm{C} 12: 0$ & 0.032 & 0.09 & 0.03 & 0.12 \\
\hline $\mathrm{C} 14: 0$ & 0.5 & 1.23 & 0.4 & 1.71 \\
\hline C14:1 & 0 & 0.01 & 0 & 0.02 \\
\hline $\mathrm{C} 15: 0$ & 0.04 & 0.1 & 0.05 & 0.1 \\
\hline C16:0 & 16.9 & 21.5 & 15.2 & 28.91 \\
\hline $\mathrm{C} 16: 1 \mathrm{n} 7$ & 0.97 & 0.88 & 0.44 & 0.46 \\
\hline $\mathrm{C} 17: 0$ & 0.09 & 0.31 & 0.15 & 0.71 \\
\hline $\mathrm{C} 17: 1 \mathrm{n} 7$ & 0.06 & 0.33 & 0.07 & 0.34 \\
\hline C18:0 & 5.46 & 21.39 & 4.85 & 19.1 \\
\hline C18:1 n9 & 30.23 & 34.23 & 26.71 & 31.45 \\
\hline $\mathrm{C} 18: 1 \mathrm{n} 11 \mathrm{t}$ & 0.09 & 0.36 & 0.09 & 0.28 \\
\hline $\mathrm{C} 18: 2 \mathrm{n} 6 \mathrm{c}$ & 42.25 & 14.78 & 48.98 & 12.99 \\
\hline C18:3 n3 & 1.02 & 1.9 & 0.23 & 1.29 \\
\hline C18:3 n6 & 0 & 0.13 & 0 & 0.11 \\
\hline C20:0 & 0.27 & 0.32 & 0.36 & 0.2 \\
\hline C20:1 n9 & 1.68 & 0.2 & 2.13 & 0 \\
\hline $\mathrm{C} 20: 2 \mathrm{n} 6$ & 0.09 & 0.62 & 0.1 & 0.5 \\
\hline $\mathrm{C} 22: 0$ & 0.15 & 0.09 & 0.11 & 0.06 \\
\hline
\end{tabular}

\section{Extraction of tissue total lipids and separation of the triacylglycerols}

Total fat content of the tissue samples was extracted according the method of FOLCH et al. (1957). Total lipids were evaporated and collected in iso-octane and ethyl-acetate (80/1 $\mathrm{vol} / \mathrm{vol}$ ). This solvent system was used to purify TAG on silicagel (230-400 mesh, 10\% water content) columns. Cholesterol was eluted with a 80/1 eluent while for TAGs a 20/1 mixture was used ( $5 \mathrm{ml}$ for $10 \mathrm{mg}$ of complex lipids). The performance of separation was controlled on Silicagel G60 plates (Merck, Darmstadt, Germany), in a developing solvent mixture of chloroform/acetone $(96 / 4 \mathrm{vol} / \mathrm{vol})$. Spots were marked by spraying primuline (5 $\mathrm{mg}$ in $100 \mathrm{~mL}$ of acetone/water [80/20, v/v]) onto the plate. 


\section{TAG hydrolysis and fractionation}

A total of $50 \mathrm{mg}$ purified TAG was subjected to extensive hydrolysis with $40 \mathrm{mg}(23.9 \mathrm{U} / \mathrm{mg})$ hog pancreatic lipase (Fluka, Buchs, Switzerland), by adding $4 \mathrm{ml} \mathrm{TRIS-HCl} \mathrm{buffer}(\mathrm{pH}=8)$ including $0.05 \% \mathrm{Na}$-deoxycholate, $0.4 \mathrm{ml} 22 \mathrm{w} / \mathrm{v} \% \mathrm{CaCl}_{2}$ and $2 \mathrm{ml} 0.1 \% \mathrm{NaCl}$ solution. The hydrolysis was performed at $40^{\circ} \mathrm{C} / 15 \mathrm{~min}$, with continuous agitation of the samples. After immediate cooling, the reaction was stopped with $2 \mathrm{ml} 4 \mathrm{M} \mathrm{HCl}$, and lipids were extracted into diethyl ether, 3 times. The organic and watery layers were separated by centrifugation $(1500 \mathrm{~g} / 3 \mathrm{~min})$, and the former was collected for evaporation $\left(30^{\circ} \mathrm{C}\right)$ for the subsequent thin layer chromatographic separation. The method was based on the procedure of LICHTFIELD (1972).

To separate hydrolysis products, silicagel G60 plates were pre-soaked in $2.3 \%$ ethanolic boric acid solution and were activated at $110^{\circ} \mathrm{C}(2 \mathrm{~h})$. Plates were developed in chloroform/ acetone $(96 / 4, v / v)$ and primuline spray was used to mark the lipid bands. The 2MAGs were scraped off the plates and were extracted into diethyl ether 3 times.

\section{Gas chromatographic fatty acid analysis}

The fatty acids (from the TAGs and the generated 2MAGs) were converted to methyl esters with Na-methoxide in methanol (CHRISTIE 1982). Gas liquid chromatography was performed on a Shimadzu 2100 apparatus (Kyoto, Japan), equipped with a capillary column ( $120 \mathrm{~m} \times 0.25 \mathrm{~mm}$ i.d., $0.20 \mu \mathrm{m}$ film, Supelco, Bellefonte, PA, USA) and flame ionization detector $\left(2 \cdot 10^{-11}\right)$. Operating conditions were as follows: injector temperature: $270^{\circ} \mathrm{C}$, detector temperature: $300^{\circ} \mathrm{C}$, helium flow: $28 \mathrm{~cm} / \mathrm{s}$. The oven temperature was graded from 80 to $205^{\circ} \mathrm{C}$ and increased by $2.5^{\circ} \mathrm{C} / \mathrm{min}, 5 \mathrm{~min}$ at $205^{\circ} \mathrm{C}$, increased from 205 to $250^{\circ} \mathrm{C}$ at $10^{\circ} \mathrm{C} / \mathrm{min}$, and $5 \mathrm{~min}$ at $250^{\circ} \mathrm{C}$. To identify individual fatty acids, an authentic fatty acid standard (Mixture Me1005, Larodan Fine Chemicals, Malmö, Sweden) was used.

Individual fatty acid proportion results were given as weight $\%$ of the total fatty acid methyl esters.

\section{Result interpretation}

The distribution frequency (\%) of individual fatty acids at the position 1,3 in the triacylglycerol molecules was calculated taking the 2MAG and TAG direct analysis data into consideration, as follows:

$$
\text { Fatty acid composition at } 1,3(\%)=\frac{\text { total }(\mathrm{TAG}) \text { composition }(\%) \cdot 3-\text { composition at } 2(\%)}{2}
$$

For the comparison of the occurrence frequency of the fatty acids at position 2 and 1,3 , the so-called positional distribution factor (PDF) was calculated for all individual fatty acids, as defined below:

$$
\text { PDF }=\log \frac{\text { composition at } 1,3(\%)}{\text { composition at } 2(\%)}
$$

Subzero PDF results refer to a high occurrence frequency at the position 2, i.e. a low abundance in the positions 1 or 3; accordingly, positive PDF values mean a frequent occurrence of a fatty acid at position 1,3. The advantage of the application of PDF is that 
this measure primarily indicates the relative distribution of fatty acids within the TAG molecule, thus giving direct information of the channeling of fatty acids.

\section{Unsaturation index and average chain length}

The unsaturation index $(\mathrm{UI})$, i.e. the number of double bonds in 100 fatty acyl chains was given as the proportion of each unsaturated fatty acid (UFA), multiplied by its double bond number. The average chain length was calculated from the multiplication of the chain length values and the respective proportions of all fatty acids.

\section{Statistical analysis}

Between-genotype differences were compared with independent-samples t-test, with the SPSS 10 (1999) software.

\section{Results}

As a general condition, the overall fatty acid composition and the fatty acid allocation order in six from seven tissues analyzed was similar, with the exception of the liver. Saturated fatty acids (SFA), in particular myristic and palmitic acids (C14:0 and C16:0) showed a predominant occurrence in the 2MAGs in all organs but the liver, where these acids were more abundant at positions 1,3. Over the chainlength of 18 (C18:0, stearic acid; C20:0, arachidic acid, C22:0, behenic acid) SFAs were only found in the 1,3 position and were basically absent in the 2MAG fraction. The allocation of odd chain SFAs (C15:0, pentadecaenoic acid, C17:0 margaric acid) was similar to those of shorter chain SFAs.

The level of unsaturation in the extrahepatic tissues was the highest at positions 1,3, followed by the TAGs, while the lowest unsaturation was measured for the 2MAGs, in both genotypes. This order was true for the total monounsaturated fatty acids (MUFA), for the total polyunsaturated fatty acids (PUFA), for the total $n 3, n 6$ and $n 9$ fatty acids, as well as for the average fatty acid chain length and for the unsaturation index. In the hepatic neutral lipids the above order was different, for all the listed fractions, namely: $2 M A G>T A G>$ fatty acid 1,3 .

In skeletal muscle (Table 2), from within the $\mathrm{n} 3$ fatty acids, the occurrence frequency of a-linolenic acid (C18:3 n3) was highest at the peripheral position (1,3). Further elongated and desaturated $\mathrm{n} 3$ acids were fully absent from the central molecular position (eicosapentaenoic acid, C20:5 n3; docosapentaenoic acid, C22:5 n3; docosahexaenoic acid, C22:6 n3). By the $n 6$ fatty acids linoleic acid (C18:2 n6), $p$-linolenic acid (C18:3 n6) and eicosadienoic (C20:2 n6) acids had positive PDF values (more frequently occupying position 1,3), dihomo- $\gamma$-linolenic acid was only detected at $s n-1,3$, while arachidonic acid (C20:4 n6) was abundant in the central molecular position. From the $\mathrm{n} 9$ fatty acids both oleic (C18:1 n9) and gondoic (C20:1 n9) acids were mostly occurring at positions 1,3. The central position was primarily occupied by palmitic, oleic and linoleic acids.

When comparing the PDF of oleic and trans vaccenic (C18:1 n11) acids (symmetric vs. asymmetric cis and trans fatty acid chains of identical length) within the TAG molecule, a genotype-associated difference was found, namely in Mangalica pigs trans vaccenic acid was more frequently found at position 1,3, while in the Hungarian Large White pigs the opposite tendency was described (Figure 1). The comparison of $\operatorname{CLA}(\mathrm{c} 9, \mathrm{t} 11)$ and linoleic 
acid distribution revealed that the trans double bond containing acyl chain tends towards the peripheral molecular position.

In the liver neutral lipids, in the $\mathrm{n} 3$ fatty acid group the allocation order of a-linolenic acid was similar to that in the muscle, while eicosatrienoic acid (C20:3 n3) had clearly defined negative PDF values (Table 3). In contrast, docosapentaenoic and docosahexaenoic acids were merely found in the 2MAGs in Mangalica pigs, while in Hungarian Large White these acids were partly detected at $s n-1,3$ in small proportions. Linoleic acid was predominant in the 2MAGs, this trait being only characteristic for the liver. In a similar way, both analyzed individual $\mathrm{n} 9$ fatty acids (oleic and gondoic acids) were significantly more frequent in the central position, i.e. in the $2 \mathrm{MAGs}$, irrespective of the genotype. The only exception, where the average fatty acid chain length was lower in the TAGs than in the 2MAGs was the Mangalica liver. For the calculated unsaturation index as well liver served as a positive exception, namely the UI values of 2 MAGs exceeded those of the fatty acids at sn-1,3.

Table 2

TAG and 2MAG fatty acid profile in the skeletal muscle of Mangalica and Hungarian Large White pigs (mean \pm SD) Triglyceride und 2-Monoglyceride Fettsäuremuster im Muskel von Mangalitza und Ungarischem Large White

\begin{tabular}{|c|c|c|c|c|c|c|c|c|c|}
\hline Fatty acid & Mangalica TAG & HLWTAG & $P N$ & Mangalica 2MAG & HLW 2 MAG & $P \quad 1$ & Mangalica PDF & HLW PDF & $P$ \\
\hline $\mathrm{C} 10: 0$ & $99 \pm 0.03$ & 0.01 & $* *$ & $19 \pm 0.003$ & $0.15 \pm 0.01$ & $* * *$ & $-0.87 \pm 0.46$ & $0.11 \pm 0.02$ & $* *$ \\
\hline 12:0 & $06 \pm 0.01$ & $0.14 \pm 0.02$ & $* * *$ & $0.17 \pm 0.01$ & $0.18 \pm 0.004$ & ns & $-1.06 \pm 0.52$ & $-0.18 \pm 0.11$ & * \\
\hline 14:0 & 10 & $1.87 \pm 0.18$ & $* * *$ & $2.73 \pm 0.08$ & $3.70 \pm 0.09$ & $* * *$ & $-1.27 \pm 0.31$ & $-0.61 \pm 0.16$ & $* *$ \\
\hline C14:1 & & $0.03=$ & $* *$ & & $0.04 \pm$ & $* *$ & & -0.02 & $* *$ \\
\hline C15:0 & $0.03 \pm 0.001$ & $0.07 \pm 0.02$ & * & $0.09 \pm 0.01$ & $0.17 \pm 0.01$ & $* * *$ & & $-0.99 \pm 0.58$ & ns \\
\hline C16:0 & $19.10 \pm 0.78$ & $25.73 \pm 0.98$ & $* * *$ & $49.20 \pm 0.28$ & $54.40 \pm 0.33$ & $* * *$ & $-1.09 \pm 0.10$ & $-0.68 \pm 0.05$ & $* *$ \\
\hline $\mathrm{C} 16: 1 \mathrm{n} 7$ & $3.97 \pm 0.66$ & $4.15 \pm 0.51$ & ns & $8.73 \pm 0.09$ & $5.55 \pm$ & $* * *$ & $-0.82 \pm 0.30$ & -0.21 & ns \\
\hline 17:0 & $0.16 \pm 0.02$ & $0.43 \pm 0.10$ & $* *$ & $0.24 \pm 0.01$ & $0.60 \pm 0.01$ & $* * *$ & $-0.33 \pm 0.15$ & $-0.27 \pm 0.16$ & ns \\
\hline C17:1 n7 & $0.22 \pm 0.02$ & $0.41 \pm$ & $* *$ & $0.34 \pm 0.04$ & $0.56 \pm 0.01$ & $* * *$ & $-0.31 \pm 0.08$ & $-0.24 \pm 0.14$ & ns \\
\hline 18:0 & & 10.14 & $* *$ & & $2.44 \pm$ & $* * *$ & $5 \pm 0.10$ & 0.04 & ns \\
\hline 18:1 n & $49.00 \pm 2.03$ & $42.14 \pm$ & $* *$ & $21.20 \pm 0.15$ & $19.20 \pm 0.25$ & $* * *$ & $0.47 \pm 0.02$ & 0.02 & ns \\
\hline $\mathrm{C} 18: 1 \mathrm{n} 11 \mathrm{t}$ & 03 & 0.17 & * & 01 & $0.06 \pm$ & $* *$ & \pm 0.18 & 0.09 & $* *$ \\
\hline LA c9t11 & & 07 & ns & & $0.04 \pm$ & * & & 0.34 & ns \\
\hline 18:2 n6 & $16.60 \pm 1.65$ & $11.44=$ & $*$ & 13.6 & $9.23 \pm 0.05$ & $* * *$ & $0.12 \pm 0.06$ & 0.11 & ns \\
\hline $18: 3$ n6 & $0.07 \pm$ & .004 & * & 0.001 & $0.07 \pm 0.002$ & $* * *$ & $0.30 \pm 0.11$ & $-0.22 \pm 0.07$ & $* * *$ \\
\hline C18:3 n3 & & & * & 0.02 & $0.39 \pm$ & $* * *$ & 0.14 & 0.02 & $* *$ \\
\hline $20: 0$ & .02 & .04 & ns & nd & nd & & na & na & \\
\hline C20:1 n9 & & 0.68 & $* *$ & $0.17 \pm 0.003$ & $0.14 \pm 0.003$ & $* * *$ & 0.15 & 0.19 & ns \\
\hline$C 20: 2$ n6 & & 09 & * & 01 & $0.23 \pm$ & $* * *$ & $0.74 \pm$ & 0.10 & $* * *$ \\
\hline$C 20: 3$ n3 & $0.20 \pm$ & .02 & * & $0.12 \pm 0.01$ & $0.31 \pm 0.01$ & $* * *$ & $0.30 \pm 0.06$ & $-0.64 \pm 0.17$ & \\
\hline C20:3 n6 & & & ns & & nd & & na & na & \\
\hline :4 n6 & 0 & .04 & ns & $0.72 \pm 0.05$ & $1.91 \pm 0.07$ & $* * *$ & $-0.11 \pm 0.12$ & na & \\
\hline C20:5 n3 & .001 & 0.001 & $* * *$ & nd & nd & & na & na & \\
\hline C22:0 & & $0.05 \pm$ & $* * *$ & nd & $0.09 \pm 0$ & $* * *$ & na & $-0.55 \pm 0.22$ & \\
\hline$: 5 \mathrm{n}$ & 2 & 0.11 & * & nd & $0.47 \pm 0.03$ & $* * *$ & na & na & \\
\hline C22:6 n3 & $0.04 \pm 0.01$ & $0.02 \pm 0.01$ & * & nd & nd & & nd & nd & \\
\hline caturat & $.70 \pm 1.75$ & $3.75 \pm 1.89$ & $* * *$ & $0 \pm 0.16$ & $0 \pm 0.30$ & $* * *$ & na & na & \\
\hline & & & $* * *$ & & & $* * *$ & & na & \\
\hline$\Sigma$ monoenoic & & & $* *$ & 30. & $25.60 \pm$ & $* * *$ & $0.03 \pm 0.00$ & $1.30 \pm 0.25$ & * \\
\hline$\Sigma$ polyunsaturated & $19.40 \pm 1.76$ & $13.66 \pm 3.25$ & $* *$ & $15.30 \pm 0.09$ & $12.70 \pm 0.10$ & $* * *$ & na & na & \\
\hline$\Sigma \mathrm{n} 3$ & & $0.98 \pm 0.04$ & * & $0.77 \pm 0.02$ & $1.17 \pm 0.03$ & $* * *$ & na & na & \\
\hline$\sum \mathrm{n} 6$ & $18.10 \pm 1.78$ & $12.56 \pm 3.20$ & $* *$ & $14.50 \pm 0.08$ & $11.40 \pm 0.11$ & $* * *$ & 11a & na & \\
\hline$\Sigma \mathrm{n} 6 / \Sigma \mathrm{n} 3$ & $16.00 \pm 2.79$ & $12.81 \pm 3.08$ & ns & $18.90 \pm 0.36$ & $9.76 \pm 0.31$ & $* * *$ & na & na & \\
\hline$\Sigma \mathrm{n} 9$ & $49.50 \pm 1.92$ & $42.82 \pm 1.72$ & $* *$ & $21.40 \pm 0.15$ & $19.40 \pm 0.25$ & $* * *$ & $1.05 \pm 0.14$ & $1.24 \pm 0.17$ & $\mathrm{~ns}$ \\
\hline$\Sigma$ unsat. $/ \Sigma$ sat. & $2.75 \pm 0.24$ & $1.59 \pm 0.13$ & $* * *$ & $0.85 \pm 0.01$ & $0.62 \pm 0.01$ & $* * *$ & na & na & \\
\hline Average chain length & $17.50 \pm 0.02$ & $17.34 \pm 0.04$ & $* * *$ & $16.70 \pm 0.00$ & $16.70 \pm 0.00$ & $* * *$ & na & na & \\
\hline Unsaturation index & $95.50 \pm 3.17$ & $77.25 \pm 5.07$ & $* * *$ & $63.50 \pm 0.09$ & $56.90 \pm 0.43$ & $* * *$ & na & na & \\
\hline
\end{tabular}

HLW Hungarian Large White, nd not detected, na not available, ns not significant, ${ }^{*} P<0.05,{ }^{* *} P<0.01,{ }^{* * *} P<0.001$ 
In the neutral lipids of the renal cortex the basic $n 3$ precursor, a-linolenic and eicosatrienoic acids showed similar allocations to the muscle, while EPA was not detected in the 2MAGs (Table 4). Only in the Mangalica pigs, DPA and DHA were totally absent in the 2MAG fraction. In the renal n6 fatty acids the basic precursor, linoleic acid had significantly different PDF values between genotypes $(0.33 \pm 0.09$ vs. $-0.07 \pm 0.08$ in Mangalica and Hungarian Large White, resp.), while also its elongated product; dihomo- $\gamma$-linolenic acid was not detected in the Mangalica renal 2MAGs. Interestingly, C20:3 n6 was fully absent in both genotypes from the 2MAGs, while arachidonic acid had positive PDF values. In the $n 9$ fatty acids, oleic acid mostly acylated at $s n-2$, while gondoic acid rather at positions 1,3 .

In the myocardial neutral lipids (Table 5), the allocation order of the fatty acids was largely similar to that in the longissimus dorsi. Small differences concerned DPA, of which the presence was only proven in the 2 MAGs of Mangalica pigs, and arachidonate, predominantly

Table 3

TAG and 2MAG fatty acid profile in the liver of Mangalica and Hungarian Large White pigs (mean \pm SD) TAG- und 2MAG-Fettsäuremuster in der Leber von Mangalitza und Ungarischem Large White

\begin{tabular}{|c|c|c|c|c|c|c|c|c|c|}
\hline Fatty acid & Mangalica TAG & HLW TAG & $P$ & Mangalica 2MAG & HLW 2MAG & $P$ & Mangalica PDF & HLW PDF & $P$ \\
\hline C10:0 & $0.02 \pm 0.001$ & $0.05 \pm 0.05$ & ns & nd & nd & & na & na & \\
\hline C12:0 & $0.05 \pm 0.003$ & $0.09 \pm 0.03$ & ns & $0.03 \pm 0.000$ & $0.04 \pm 0.000$ & $* * *$ & $0.38 \pm 0.03$ & $0.45 \pm 0.16$ & ns \\
\hline C14:0 & $1.22 \pm 0.43$ & $1.37 \pm 0.34$ & ns & $0.81 \pm 0.03$ & $0.99 \pm 0.002$ & $* *$ & $0.22 \pm 0.22$ & $0.19 \pm 0.15$ & ns \\
\hline C14:1 & $0.01 \pm 0.01$ & $0.01 \pm 0.01$ & ns & nd & nd & & na & na & \\
\hline C15:0 & $0.29 \pm 0.04$ & $0.21 \pm 0.10$ & ns & $0.09 \pm 0.002$ & $0.19 \pm 0.002$ & $* * *$ & $0.64 \pm 0.05$ & $-0.08 \pm 0.45$ & ns \\
\hline C16:0 & $24.68 \pm 0.78$ & $23.85 \pm 4.58$ & ns & $14.49 \pm 0.23$ & $18.92 \pm 0.01$ & $* * *$ & $0.31 \pm 0.01$ & $0.13 \pm 0.11$ & ns \\
\hline $\mathrm{C} 16: 1 \mathrm{n} 7$ & $2.58 \pm 0.18$ & $1.06 \pm 0.58$ & ns & $2.75 \pm 0.02$ & $1.89 \pm 0.01$ & $* * *$ & $-0.04 \pm 0.05$ & $-0.28 \pm 0.25$ & ns \\
\hline C17:0 & $1.29 \pm 0.17$ & $1.21 \pm 0.43$ & ns & $0.43 \pm 0.01$ & $0.53 \pm 0.003$ & $* * *$ & $0.60 \pm 0.08$ & $0.44 \pm 0.22$ & ns \\
\hline $\mathrm{C} 17: 1 \mathrm{n} 7$ & $0.37 \pm 0.16$ & $0.43 \pm 0.13$ & $*$ & $0.42 \pm 0.01$ & $0.48 \pm 0.002$ & $* * *$ & $-0.13 \pm 0.31$ & $-0.10 \pm 0.19$ & ns \\
\hline C18:0 & $10.19 \pm 0.82$ & $14.08 \pm 4.5$ & ns & $3.50 \pm 0.02$ & $3.12 \pm 0.004$ & $* * *$ & $0.59 \pm 0.04$ & $0.78 \pm 0.15$ & ns \\
\hline C18:1 n9c & $31.96 \pm 0.83$ & $31.09 \pm 0.56$ & ns & $40.55 \pm 0.06$ & $38.92 \pm 0.02$ & $* * *$ & $-0.17 \pm 0.02$ & $-0.16 \pm 0.01$ & ns \\
\hline $\mathrm{C} 18: 1 \mathrm{n} 11 \mathrm{t}$ & $0.29 \pm 0.05$ & $0.51 \pm 0.21$ & ns & $0.15 \pm 0.01$ & $0.09 \pm 0.002$ & $* * *$ & $0.36 \pm 0.11$ & $0.89 \pm 0.21$ & ns \\
\hline CLA c9t11 & $0.12 \pm 0.03$ & $0.23 \pm 0.02$ & ns & $0.11 \pm 0.01$ & $0.19 \pm 0.001$ & $* * *$ & $0.03 \pm 0.24$ & $0.11 \pm 0.05$ & ns \\
\hline C18:2 n6 & $16.98 \pm 2.06$ & $16.45 \pm 3.05$ & * & $24.78 \pm 0.03$ & $23.35 \pm 0.02$ & $* * *$ & $-0.28 \pm 0.10$ & $-0.28 \pm 0.18$ & ns \\
\hline C18:3 n6 & 0.57 & $0.37 \pm$ & ns & $0.41 \pm 0.00$ & $0.23 \pm 0.003$ & $* * *$ & $0.19 \pm 0.17$ & $0.07 \pm 0.63$ & ns \\
\hline C18:3 n3 & $0.61 \pm 0.11$ & $0.93 \pm$ & ns & $0.22 \pm 0.01$ & $0.29 \pm 0.002$ & $* *$ & $0.55 \pm 0.08$ & $0.58 \pm 0.29$ & ns \\
\hline C20:0 & $0.15=$ & $0.18 \pm$ & ns & $0.03 \pm 0.01$ & $0.03 \pm 0.003$ & - & $0.90 \pm 0.24$ & $0.91 \pm 0.06$ & ns \\
\hline C20:1 n9 & 0.41 & $0.54=$ & ns & $0.55 \pm 0.00$ & $0.45 \pm$ & $* * *$ & $-0.23 \pm 0.13$ & $0.48 \pm 0.001$ & ns \\
\hline$C 20: 2$ n6 & $0.73 \pm 0.1$ & $0.41 \pm 0.35$ & ns & $0.62 \pm 0.00$ & $0.71 \pm 0.003$ & $* * *$ & $0.10 \pm 0.09$ & $-0.12 \pm 0.17$ & ns \\
\hline$C 20: 3$ n3 & $0.94 \pm 0.1$ & $0.80 \pm$ & ns & $1.39 \pm 0.03$ & $1.25 \pm 0.005$ & $* *$ & $-0.29 \pm 0.07$ & $-0.17 \pm 0.18$ & ns \\
\hline C20:3 n6 & 0.02 & $0.10 \pm$ & ns & $0.05 \pm 0.01$ & $0.05 \pm 0.000$ & - & $0.53 \pm 0.01$ & $0.29 \pm 0.36$ & ns \\
\hline C20:4 n6 & $5.48 \pm 0.63$ & $5.11 \pm 3.0$ & ns & $6.36 \pm 0.02$ & $6.11 \pm 0.01$ & $* *$ & $-0.10 \pm 0.08$ & $0.07 \pm 0.01$ & ns \\
\hline$C 20: 5 n 3$ & $0.14 \pm 0.02$ & $0.16 \pm 0.17$ & ns & $0.08 \pm 0.00$ & $0.16 \pm 0.002$ & $* * *$ & $0.36 \pm 0.07$ & $0.09 \pm 0.50$ & ns \\
\hline C22:0 & $0.18 \pm 0.06$ & $0.28 \pm 0.2$ & ns & $0.13 \pm 0.00$ & $0.36 \pm 0.004$ & $* * *$ & $0.19 \pm 0.19$ & $0.05 \pm 0.02$ & ns \\
\hline $\mathrm{C} 22: 5 \mathrm{n} 3$ & $0.35 \pm 0.04$ & $0.58 \pm 0.37$ & ns & $1.14 \pm 0.03$ & $1.54 \pm 0.01$ & $* * *$ & na & $-0.67 \pm 0.40$ & \\
\hline $\mathrm{C} 22: 6 \mathrm{n} 3$ & $0.30 \pm 0.08$ & $0.07 \pm 0.02$ & $*$ & $0.93 \pm 0.03$ & $0.11 \pm 0.01$ & $* * *$ & $-1.29 \pm 0.001$ & $-0.53 \pm 0.57$ & ns \\
\hline satur & 30.0 & J2. & ns & & $24.19 \pm$ & $* * *$ & na & na & \\
\hline$\sum$ unsaturated & $61.95 \pm 0.08$ & $58.68 \pm 8.4$ & ns & $80.50 \pm 0.20$ & $75.81 \pm 0.01$ & $* * *$ & na & na & \\
\hline$\Sigma$ monoenoic & $35.61 \pm 1.17$ & $33.46 \pm 1.66$ & ns & $44.42 \pm 0.04$ & $41.83 \pm 0.03$ & * & na & na & \\
\hline$\Sigma$ polyunsaturated & $26.34 \pm 1.08$ & $25.22 \pm 6.75$ & ns & $36.08 \pm 0.16$ & $33.98 \pm 0.02$ & $* * *$ & na & $0.46 \pm 0.07$ & \\
\hline$\Sigma \mathrm{n} 3$ & $2.34 \pm 0.35$ & $2.54 \pm 0.55$ & ns & $3.75 \pm 0.10$ & $3.35 \pm 0.01$ & $* *$ & na & $-0.48 \pm 0.11$ & \\
\hline$\sum n 6$ & $23.89 \pm 1.47$ & $22.45 \pm 6.2$ & ns & $32.22 \pm 0.06$ & $30.44 \pm 0.01$ & $* * *$ & $0.43 \pm 0.10$ & $0.79 \pm 0.21$ & ns \\
\hline$\sum n 6 / \sum n 3$ & $10.38 \pm 2.17$ & $8.75 \pm 0.81$ & ns & $8.59 \pm 0.22$ & $9.10 \pm 0.02$ & $*$ & na & $-1.96 \pm 0.17$ & \\
\hline$\Sigma \mathrm{n} 9$ & $32.36 \pm 0.77$ & $31.45 \pm 1.01$ & ns & $41.11 \pm 0.06$ & $39.37 \pm 0.03$ & $* * *$ & $-0.40 \pm 0.11$ & $0.33 \pm 0.05$ & ns \\
\hline$\Sigma$ unsat./ $\Sigma$ sat. & $1.63 \pm 0.01$ & $1.48 \pm 0.45$ & ns & $4.13 \pm 0.05$ & $3.13 \pm 0.00$ & $* * *$ & na & na & \\
\hline average chain I & $17.57 \pm 0.05$ & $17.59 \pm 0.19$ & ns & $17.88 \pm 0.01$ & $17.78 \pm 0.003$ & $* * *$ & na & na & \\
\hline unsaturation index & $103.45 \pm 0.72$ & $98.01 \pm 22.36$ & ns & $138.34 \pm 0.67$ & $128.55 \pm 0.04$ & $* * *$ & na & na & \\
\hline
\end{tabular}

HLW Hungarian Large White, nd not detected, na not available, ns not significant, ${ }^{*} P<0.05,{ }^{* *} P<0.01,{ }^{* * *} P<0.001$ 
acylating at positions 1,3 . Interestingly, DHA was not detected in the 2 MAGs, only in trace amounts in the 1,3 position. Moreover, conjugated linoleic acid (C18:2 c9t11) was found to be exclusively accumulated at the positions 1,3 in the myocardium.

In the adipose tissue the mostly important genotype dependent difference was found in the distribution frequency of palmitic acid, namely in Mangalica the total palmitate moiety was found in the 2MAGs, while in the meat-type pigs a smaller proportion was also detected in the 1,3 position (Table 6 ). Adipose tissue was the only site where from the identically long C18:1 fatty acids (C18:1 $\mathrm{n} 9$ vs. C18:1 n11) the symmetric form had the higher PDF values in both genotypes (Figure 1).

In the n6 fatty acid group a further genotype-dependent difference was found: in Mangalica the PDF values of archidonic acid largely exceeded the of linoleic acid, while the opposite was measured in the Hungarian Large White pigs (Figure 2).

Table 4

TAG and 2 MAG fatty acid profile in the renal cortex of Mangalica and Hungarian Large White pigs (mean $\pm S D$ ) TAG- und 2MAG-Fettsäuremuster der Niere von Mangalitza und Ungarischem Large White

\begin{tabular}{|c|c|c|c|c|c|c|c|c|c|}
\hline Fatty acid & Mangalica TAG & HLW TAG & $P 1$ & Mangalica 2MAG & HLW 2 MAG & $P$ & Mangalica PDF & HLW PDF & $P$ \\
\hline C10:0 & $0.02 \pm 0.01$ & $0.03 \pm 0.01$ & ns & $0.03 \pm 0.004$ & nd & ns & $0.01 \pm 0.07$ & na & ns \\
\hline C12:0 & $0.05 \pm 0.01$ & $0.14 \pm 0.01$ & $* *$ & $0.08 \pm 0.004$ & $0.08 \pm 0.001$ & $* * *$ & $-0.37 \pm 0.20$ & $0.32 \pm 0.06$ & ns \\
\hline C14:0 & $1.22 \pm 0.31$ & $2.50 \pm 0.13$ & $* *$ & $2.19 \pm 0.03$ & $2.70 \pm 0.02$ & $* * *$ & $-0.56 \pm 0.37$ & $-0.05 \pm 0.04$ & 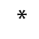 \\
\hline C14:1 & nd & $0.00 \pm 0.01$ & $* *$ & nd & nd & & na & na & \\
\hline C15:0 & $0.10 \pm 0.05$ & $0.43 \pm 0.11$ & ns & $0.14 \pm 0.02$ & $0.52 \pm 0.02$ & $* * *$ & $-0.55 \pm 0.76$ & $-0.15 \pm 0.19$ & ns \\
\hline C16:0 & $31.40 \pm 7.05$ & $36.76 \pm 1.88$ & ns & $65.60 \pm 0.16$ & $53.95 \pm 0.34$ & $* * *$ & $-0.76 \pm 0.38$ & $-0.28 \pm 0.05$ & $*$ \\
\hline $\mathrm{C} 16: 1 \mathrm{n} 7$ & $1.46 \pm 0.32$ & $1.75 \pm 0.80$ & $* *$ & $2.73 \pm 0.07$ & $1.35 \pm 0.01$ & $* * *$ & $-0.62 \pm 0.40$ & $0.10 \pm 0.25$ & * \\
\hline C17:0 & $0.48 \pm 0.08$ & $1.05 \pm 0.19$ & $*$ & $0.62 \pm 0.004$ & $0.57 \pm 0.19$ & $* * *$ & $-0.18 \pm 0.13$ & $0.38 \pm 0.19$ & $* *$ \\
\hline $\mathrm{C} 17: 1 \mathrm{n} 7$ & $0.16 \pm 0.07$ & $0.23 \pm 0.09$ & ns & $0.27 \pm 0.01$ & $0.23 \pm 0.01$ & $* *$ & $-0.60 \pm 0.46$ & $-0.09 \pm 0.30$ & ns \\
\hline C18:0 & $12.76 \pm 1.15$ & $11.10 \pm 0.82$ & ns & $3.28 \pm 0.04$ & $2.14 \pm 0.01$ & $* * *$ & $0.73 \pm 0.05$ & $0.86 \pm 0.03$ & $*$ \\
\hline C18:1 n9c & $35.65 \pm 7.52$ & $20.80 \pm 4.15$ & $*$ & $17.05 \pm 0.05$ & $16.81 \pm 0.05$ & $* *$ & $0.41 \pm 0.11$ & $0.12 \pm 0.11$ & $* *$ \\
\hline $\mathrm{C} 18: 1 \mathrm{n} 11 \mathrm{t}$ & $0.20 \pm 0.05$ & $0.31 \pm 0.06$ & ns & $0.06 \pm 0.01$ & $0.12 \pm 0.01$ & $* * *$ & $0.61 \pm 0.18$ & $0.51 \pm 0.14$ & ns \\
\hline CLA c9t11 & $0.09 \pm 0.005$ & $0.13 \pm 0.06$ & ns & nd & $0.07 \pm 0.002$ & ns & na & $0.28 \pm 0.28$ & ns \\
\hline C18:2 n6 & $11.99 \pm 2.04$ & $14.17 \pm 1.62$ & ns & $6.77 \pm 0.06$ & $15.62 \pm 0.10$ & $* * *$ & $0.33 \pm 0.09$ & $-0.07 \pm 0.08$ & $* *$ \\
\hline C18:3 n6 & $0.04 \pm 0.02$ & $0.14 \pm 0.05$ & $*$ & nd & $0.09 \pm 0.01$ & ns & na & $0.25 \pm 0.14$ & ns \\
\hline C18:3 n3 & $1.17 \pm 0.19$ & $1.67 \pm 0.90$ & ns & $0.15 \pm 0.01$ & $0.23 \pm 0.003$ & $* * *$ & $1.04 \pm 0.05$ & $0.91 \pm 0.42$ & ns \\
\hline $\mathrm{C} 20: 0$ & $0.28 \pm 0.14$ & $0.57 \pm 0.08$ & $*$ & nd & nd & ns & na & na & \\
\hline C20:1 n9 & $0.50 \pm 0.05$ & $0.36 \pm 0.72$ & ns & $0.22 \pm 0.01$ & $0.54 \pm 0.00$ & ns & $0.47 \pm 0.09$ & $0.55 \pm 0.13$ & ns \\
\hline C20:2 n6 & $0.94 \pm 0.19$ & $2.01 \pm 0.72$ & ns & $0.22 \pm 0.03$ & $0.83 \pm 0.01$ & $* * *$ & $0.76 \pm 0.15$ & $0.47 \pm 0.19$ & ns \\
\hline$C 20: 3$ n3 & $0.25 \pm 0.14$ & $1.48 \pm 0.81$ & ns & $0.12 \pm 0.03$ & $0.69 \pm 0.40$ & ns & $0.36 \pm 0.27$ & $0.53 \pm 0.48$ & ns \\
\hline C20:3 n6 & $0.18 \pm 0.09$ & $0.41 \pm 0.21$ & ns & nd & nd & ns & na & na & \\
\hline C20:4 n6 & $1.00 \pm 0.53$ & $2.60 \pm 0.67$ & $*$ & $0.45 \pm 0.03$ & $2.06 \pm 0.03$ & $* * *$ & $0.36 \pm 0.42$ & $0.12 \pm 0.15$ & ns \\
\hline$C 20: 5 n 3$ & $0.02 \pm 0.001$ & $0.12 \pm 0.13$ & ns & nd & nd & ns & na & & \\
\hline C22:0 & $0.08 \pm 0.06$ & $0.12 \pm 0.10$ & ns & nd & $0.05 \pm 0.01$ & ns & na & $0.44 \pm 0.36$ & \\
\hline $\mathrm{C} 22: 5 \mathrm{n} 3$ & $0.07 \pm 0.01$ & $0.82 \pm 0.48$ & $*$ & nd & $1.06 \pm 0.05$ & ns & na & $-0.58 \pm 0.89$ & \\
\hline $\mathrm{C} 22: 6 \mathrm{n} 3$ & $0.10 \pm 0.04$ & $0.29 \pm 0.37$ & ns & nd & $0.30 \pm 0.02$ & ns & na & $-0.51 \pm 1.35$ & \\
\hline$\Sigma$ satur & 46.40 & 52.7 & ns & 71. & $00.00 I$ & . & $\pi d$ & na & \\
\hline$\Sigma$ unsaturated & $53.60 \pm 8.73$ & $47.30 \pm 2.49$ & ns & $28.06 \pm 0.09$ & $40.00 \pm 0.45$ & $* * *$ & na & na & \\
\hline$\Sigma$ monoenoic & $37.79 \pm 7.62$ & $23.45 \pm 4.70$ & ns & $20.34 \pm 0.01$ & $19.05 \pm 0.05$ & $* * *$ & na & na & \\
\hline$\Sigma$ polyunsaturated & $15.80 \pm 1.11$ & $23.84 \pm 4.80$ & ns & $7.71 \pm 0.09$ & $20.95 \pm 0.44$ & $* * *$ & na & na & \\
\hline$\Sigma \mathrm{n} 3$ & $1.56 \pm 0.20$ & $4.38 \pm 2.45$ & ns & $0.27 \pm 0.03$ & $2.28 \pm 0.35$ & $* * *$ & na & na & \\
\hline$\Sigma \mathrm{n} 6$ & $14.16 \pm 1.27$ & $19.33 \pm 2.34$ & ns & $7.44 \pm 0.11$ & $18.60 \pm 0.14$ & $* * *$ & na & na & \\
\hline$\sum n 6 / \Sigma n 3$ & $9.21 \pm 1.79$ & $5.42 \pm 2.61$ & ns & $27.95 \pm 3.11$ & $8.34 \pm 1.49$ & $* * *$ & na & na & \\
\hline$\sum \mathrm{n} 9$ & $35.98 \pm 7.29$ & $21.16 \pm 4.79$ & ns & $17.27 \pm 0.05$ & $17.34 \pm 0.05$ & ns & na & na & \\
\hline$\Sigma$ unsat./ $\Sigma$ sat. & $1.21 \pm 0.42$ & $0.90 \pm 0.09$ & ns & $0.39 \pm 0.00$ & $0.67 \pm 0.01$ & $* * *$ & $0.83 \pm 0.18$ & $0.81 \pm 0.23$ & ns \\
\hline average chain length & $17.35 \pm 0.12$ & $17.29 \pm 0.07$ & ns & $16.55 \pm 0.00$ & $16.90 \pm 0.02$ & $* * *$ & na & na & \\
\hline unsaturation index & $73.52 \pm 8.41$ & $83.43 \pm 9.54$ & ns & $36.94 \pm 0.25$ & $70.47 \pm 1.24$ & $* * *$ & na & na & \\
\hline
\end{tabular}

HLW Hungarian Large White, nd not detected, na not available, ns not significant, ${ }^{*} P<0.05$, ${ }^{* *} P<0.01,{ }^{* * *} P<0.001$ 
Table 5

TAG and 2MAG fatty acid profile in the myocardium of Mangalica and Hungarian Large White pigs (mean \pm SD) TAG- und 2MAG-Fettsäuremuster im Herzmuskel vom Mangalitza und Ungarischem Large White

\begin{tabular}{|c|c|c|c|c|c|c|c|c|c|}
\hline Fatty acid & Mangalica TAG & HLW TAG & $P$ & Mangalica 2MAG & HLW 2MAG & $P$ & Mangalica PDF & HLW PDF & $P$ \\
\hline C10:0 & $0.04 \pm 0.01$ & $0.09 \pm 0.02$ & ns & $0.05 \pm 0.01$ & $0.09 \pm 0.00$ & $* * *$ & $-0.16 \pm 0.18$ & $-0.13 \pm 0.07$ & ns \\
\hline C12:0 & $0.04 \pm 0.01$ & $0.11 \pm 0.01$ & $* * *$ & $0.08 \pm 0.003$ & $0.18 \pm 0.001$ & $* * *$ & $-0.72 \pm 0.20$ & $-0.19 \pm 0.25$ & $*$ \\
\hline C $14: 0$ & $0.64 \pm 0.08$ & $1.38 \pm 0.11$ & $* * *$ & $\quad 1.83 \pm 0.03$ & $3.20 \pm 0.01$ & $* * *$ & $-1.34 \pm 0.22$ & $-0.46 \pm 0.50$ & * \\
\hline C14:1 & $0.01 \pm 0.001$ & $0.01 \pm 0.01$ & ns & $0.02 \pm 0.004$ & nd & & $-0.49 \pm 0.02$ & na & \\
\hline C15:0 & $0.05 \pm 0.005$ & $0.08 \pm 0.01$ & $* *$ & $0.13 \pm 0.02$ & $0.20 \pm 0.01$ & $* * *$ & $-0.75 \pm 0.34$ & $-0.56 \pm 0.60$ & ns \\
\hline C16:0 & $18.50 \pm 0.83$ & $26.15 \pm 1.57$ & $* * *$ & $54.56 \pm 0.18$ & $62.34 \pm 0.22$ & $* * *$ & $-1.70 \pm 0.08$ & $-0.52 \pm 0.55$ & $* *$ \\
\hline $\mathrm{C} 16: 1 \mathrm{n} 7$ & $2.43 \pm 0.10$ & $2.11 \pm 0.23$ & $* *$ & $4.67 \pm 0.13$ & $3.55 \pm 0.05$ & $* * *$ & $-0.55 \pm 0.04$ & $-0.26 \pm 0.32$ & ns \\
\hline C17:0 & $0.37 \pm 0.04$ & $0.63 \pm 0.07$ & * & $0.67 \pm 0.01$ & $0.79 \pm 0.01$ & $* * *$ & $-0.50 \pm 0.13$ & $-0.08 \pm 0.14$ & $* *$ \\
\hline C17:1 n7 & $0.22 \pm 0.08$ & $0.38 \pm 0.06$ & $* *$ & $0.40 \pm 0.01$ & $0.32 \pm 0.28$ & ns & $-0.41 \pm 0.25$ & $0.00 \pm 0.06$ & ns \\
\hline C18:0 & $9.70 \pm 0.30$ & $16.23 \pm 2.11$ & $* *$ & $3.59 \pm 0.09$ & $3.37 \pm 0.02$ & $*$ & $0.55 \pm 0.03$ & $0.84 \pm 0.07$ & $* *$ \\
\hline C18:1 n9c & $42.28 \pm 1.90$ & $36.35 \pm 2.87$ & $* *$ & $20.67 \pm 0.19$ & $16.96 \pm 0.05$ & $* * *$ & $0.41 \pm 0.03$ & $0.14 \pm 0.49$ & ns \\
\hline $\mathrm{C} 18: 1 \mathrm{n} 11 \mathrm{t}$ & $0.21 \pm 0.07$ & $0.19 \pm 0.08$ & ns & $0.16 \pm 0.01$ & $0.03 \pm 0.001$ & $* * *$ & $0.14 \pm 0.26$ & $1.00 \pm 0.19$ & $*$ \\
\hline CLA c9t11 & $0.13 \pm 0.01$ & $0.08 \pm 0.04$ & ns & nd & nd & & na & na & \\
\hline C18:2 n6 & $19.43 \pm 1.42$ & $12.23 \pm 1.15$ & $* *$ & $11.59 \pm 0.12$ & $7.44 \pm 0.02$ & $* * *$ & $0.30 \pm 0.04$ & $0.10 \pm 0.29$ & ns \\
\hline C18:3 n6 & $0.07 \pm 0.01$ & $0.09 \pm 0.03$ & $* *$ & $0.03 \pm 0.00$ & $0.02 \pm 0.00$ & * & $0.46 \pm 0.13$ & $0.67 \pm 0.21$ & ns \\
\hline C18:3 n3 & $0.92 \pm 0.45$ & $1.07 \pm 0.67$ & ns & $0.41 \pm 0.01$ & $0.27 \pm 0.01$ & $* * *$ & $0.40 \pm 0.27$ & $0.48 \pm 0.61$ & ns \\
\hline C20:0 & $0.14 \pm 0.02$ & $0.21 \pm 0.08$ & ns & nd & nd & & na & na & \\
\hline C20:1 n9 & $0.64 \pm 0.33$ & $1.10 \pm 0.23$ & ns & $0.19 \pm 0.005$ & $0.18 \pm 0.01$ & ns & $0.58 \pm 0.33$ & $0.55 \pm 0.54$ & ns \\
\hline $\mathrm{C} 20: 2 \mathrm{n} 6$ & $0.76 \pm 0.06$ & $0.60 \pm 0.03$ & ns & $0.17 \pm 0.003$ & $0.22 \pm 0.01$ & $* * *$ & $0.78 \pm 0.04$ & $0.19 \pm 0.66$ & ns \\
\hline C20:3 n3 & $0.20 \pm 0.03$ & $0.29 \pm 0.02$ & $* *$ & $0.08 \pm 0.004$ & $0.15 \pm 0.01$ & $* * *$ & $0.47 \pm 0.08$ & $0.20 \pm 0.36$ & ns \\
\hline C20:3 n6 & $0.07 \pm 0.02$ & $0.09 \pm 0.03$ & ns & nd & nd & & na & na & \\
\hline C20:4 n6 & $3.16 \pm 3.93$ & $0.99 \pm 0.11$ & ns & $0.57 \pm 0.03$ & $0.64 \pm 0.04$ & * & $0.63 \pm 0.53$ & $0.13 \pm 0.23$ & ns \\
\hline C20:5 n3 & $0.01 \pm 0.01$ & $0.04 \pm 0.02$ & ns & nd & nd & & na & na & \\
\hline $\mathrm{C} 22: 0$ & $0.03 \pm 0.01$ & $0.06 \pm 0.01$ & $* *$ & nd & $0.03 \pm 0.003$ & & na & $0.18 \pm 0.31$ & \\
\hline $\mathrm{C} 22: 5 \mathrm{n} 3$ & $0.09 \pm 0.02$ & $0.17 \pm 0.04$ & $*$ & $0.11 \pm 0.004$ & nd & & $-0.17 \pm 0.14$ & na & \\
\hline $\mathrm{C} 22: 6 \mathrm{n} 3$ & $0.05 \pm 0.002$ & $0.03 \pm 0.01$ & * & nd & nd & & na & na & \\
\hline$\Sigma$ saturated & $29.51 \pm 0.77$ & $44.93 \pm 3.72$ & $* * *$ & $60.92 \pm 0.15$ & $70.21 \pm 0.21$ & $* * *$ & na & na & \\
\hline$\sum$ unsaturated & $70.49 \pm 0.77$ & $55.07 \pm 3.72$ & $* * *$ & $39.08 \pm 0.15$ & $29.79 \pm 0.20$ & $* * *$ & na & na & \\
\hline$\Sigma$ monoenoic & $45.62 \pm 1.76$ & $39.41 \pm 2.40$ & $*$ & $26.11 \pm 0.26$ & $21.04 \pm 0.25$ & $* * *$ & na & na & \\
\hline$\Sigma$ polyunsaturated & $24.87 \pm 2.50$ & $15.66 \pm 1.41$ & $* *$ & $12.97 \pm 0.14$ & $8.75 \pm 0.06$ & $* * *$ & na & na & \\
\hline$\sum \mathrm{n} 3$ & $1.25 \pm 0.49$ & $1.58 \pm 0.62$ & ns & $0.61 \pm 0.003$ & $0.42 \pm 0.01$ & $* * *$ & na & na & \\
\hline$\Sigma \mathrm{n} 6$ & $23.49 \pm 2.74$ & $14.00 \pm 1.09$ & $* *$ & $12.36 \pm 0.14$ & $8.33 \pm 0.05$ & $* * *$ & na & na & \\
\hline$\Sigma \mathrm{n} 6 / \Sigma \mathrm{n} 3$ & $21.37 \pm 9.71$ & $9.97 \pm 4.37$ & ns & $20.23 \pm 0.13$ & $19.73 \pm 0.28$ & * & na & na & \\
\hline$\sum \mathrm{n} 9$ & $42.76 \pm 1.68$ & $36.72 \pm 2.24$ & $* *$ & $20.86 \pm 0.19$ & $17.15 \pm 0.06$ & $* * *$ & $0.92 \pm 0.41$ & $0.53 \pm 1.11$ & ns \\
\hline$\Sigma$ unsat./ $\Sigma$ sat. & $2.39 \pm 0.09$ & $1.24 \pm 0.18$ & $* * *$ & $0.64 \pm 0.004$ & $0.42 \pm 0.004$ & $* * *$ & na & na & \\
\hline Average chain length & $17.64 \pm 0.10$ & $17.42 \pm 0.02$ & $* *$ & $16.74 \pm 0.003$ & $16.54 \pm 0.002$ & $* * *$ & na & na & \\
\hline Unsaturation index & $103.36 \pm 10.71$ & $74.76 \pm 5.38$ & $* *$ & $54.06 \pm 0.19$ & $40.27 \pm 0.17$ & $* * *$ & na & na & \\
\hline
\end{tabular}

HLW Hungarian Large White, nd not detected, na not available, ns not significant, ${ }^{*} P<0.05,{ }^{* *} P<0.01,{ }^{* * *} P<0.001$

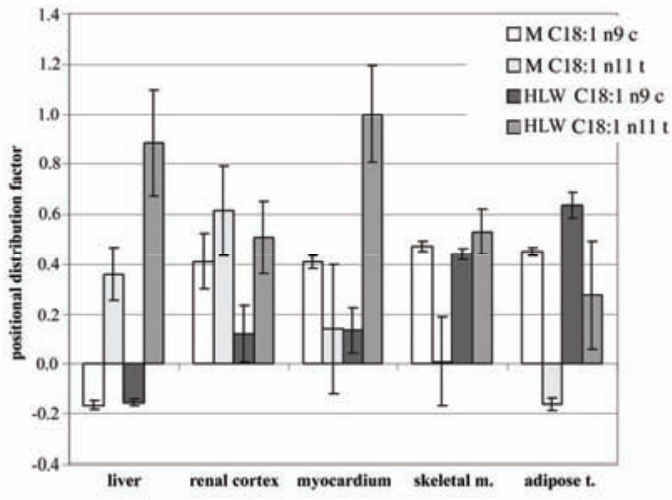

Figure1

The positional distribution of identically long C18 monounsaturated fatty acids of different molecular symmetry and double bond conformation mit differenter molekulare Symmetrie 
Table 6

TAG and 2MAG fatty acid profile in the adipose tissue of Mangalica and Hungarian Large White pigs (mean \pm SD) TAG- und 2MAG-Fettsäuremuster des Rückenfettes von Mangalitza und Ungarischem Large White

\begin{tabular}{|c|c|c|c|c|c|c|c|c|c|}
\hline Fatty acid & Mangalica TAG & HLW TAG & $P$ & Mangalica 2MAG & HLW $2 M A G$ & $P$ & Mangalica PDF & HLW PDF & $P$ \\
\hline $\mathrm{C} 10: 0$ & $0.06 \pm 0.01$ & $0.12 \pm 0.02$ & $* *$ & $0.07 \pm 0.003$ & $0.13 \pm 0.01$ & $* * *$ & $-0.06 \pm 0.10$ & $-0.03 \pm 0.11$ & ns \\
\hline C12:0 & $0.07 \pm 0.01$ & $0.10 \pm 0.01$ & $*$ & $0.12 \pm 0.01$ & $0.20 \pm 0.01$ & $* * *$ & $-0.47 \pm 0.20$ & $-0.67 \pm 0.21$ & ns \\
\hline C14:0 & $1.30 \pm 0.10$ & $1.61 \pm 0.14$ & * & $3.37 \pm 0.11$ & $4.10 \pm 0.12$ & $* * *$ & $-1.16 \pm 0.25$ & $-1.08 \pm 0.17$ & ns \\
\hline C14:1 & $0.02 \pm 0.002$ & $0.03 \pm 0.01$ & ns & $0.04 \pm 0.02$ & $0.04 \pm 0.004$ & ns & $-0.31 \pm 0.12$ & $-0.29 \pm 0.31$ & ns \\
\hline C15:0 & $0.03 \pm 0.003$ & $0.05 \pm 0.02$ & ns & $0.10 \pm 0.02$ & $0.12 \pm 0.01$ & $* *$ & & $-0.72 \pm 0.31$ & \\
\hline C16:0 & $18.56 \pm 1.92$ & $24.41 \pm 0.70$ & $* *$ & $56.38 \pm 1.40$ & $66.62 \pm 0.57$ & $* * *$ & $-1.58 \pm 0.41$ & $-1.32 \pm 0.15$ & ns \\
\hline $\mathrm{C} 16: 1 \mathrm{n} 7$ & $2.60 \pm 0.30$ & $3.58 \pm 0.87$ & ns & $4.46 \pm 0.17$ & $6.36 \pm 0.35$ & $* * *$ & $-0.44 \pm 0.12$ & $-0.54 \pm 0.34$ & ns \\
\hline C17:0 & $0.17 \pm 0.02$ & $0.36 \pm 0.17$ & ns & $0.34 \pm 0.01$ & $0.47 \pm 0.05$ & $* *$ & $-0.63 \pm 0.11$ & $-0.44 \pm 0.67$ & ns \\
\hline C17:1 n7 & $0.20 \pm 0.01$ & $0.39 \pm 0.13$ & $* * *$ & $0.35 \pm 0.04$ & $0.52 \pm 0.01$ & $* * *$ & $-0.44 \pm 0.09$ & $-0.33 \pm 0.45$ & ns \\
\hline C18:0 & $6.74 \pm 1.18$ & $9.38 \pm 1.17$ & ns & $2.44 \pm 0.08$ & $2.19 \pm 0.08$ & $* *$ & $0.56 \pm 0.09$ & $0.77 \pm 0.06$ & ** \\
\hline C18:1 n9c & $45.84 \pm 0.98$ & $45.32 \pm 4.89$ & ns & $20.69 \pm 0.85$ & $14.05 \pm 0.43$ & $* * *$ & $0.45 \pm 0.01$ & $0.64 \pm 0.05$ & $* * *$ \\
\hline $\mathrm{C} 18: 1 \mathrm{n} 11 \mathrm{t}$ & $0.17 \pm 0.02$ & $0.18 \pm 0.04$ & ns & $0.21 \pm 0.06$ & $0.12 \pm 0.04$ & $* *$ & $-0.16 \pm 0.27$ & $0.28 \pm 0.22$ & $* *$ \\
\hline CLA c9t11 & $0.16 \pm 0.02$ & $0.15 \pm 0.05$ & * & $0.03 \pm 0.00$ & $0.02 \pm 0.00$ & $* * *$ & $0.95 \pm 0.06$ & $0.95 \pm 0.02$ & ns \\
\hline C18:2 n6 & $20.12 \pm 2.45$ & $11.60 \pm 4.83$ & ns & $10.53 \pm 0.88$ & $4.39 \pm 0.42$ & $* *$ & $0.37 \pm 0.07$ & $0.49 \pm 0.23$ & ns \\
\hline C18:3 n6 & $0.04 \pm 0.01$ & $0.03 \pm 0.01$ & $* *$ & nd & $0.00 \pm 0.00$ & & $0.66 \pm 0.05$ & $0.57 \pm 0.13$ & ns \\
\hline C18:3 n3 & $1.11 \pm 0.12$ & $0.69 \pm 0.10$ & ns & $0.33 \pm 0.02$ & $0.24 \pm 0.03$ & $* * *$ & na & na & \\
\hline C20:0 & $0.13 \pm 0.03$ & $0.16 \pm 0.04$ & ns & nd & nd & & na & na & \\
\hline C20:1 n9 & $0.58 \pm 0.09$ & $0.76 \pm 0.29$ & $* * *$ & $0.20 \pm 0.02$ & $0.13 \pm 0.01$ & $* * *$ & $0.59 \pm 0.08$ & $0.91 \pm 0.19$ & * \\
\hline $\mathrm{C} 20: 2 \mathrm{n} 6$ & $1.36 \pm 0.07$ & $0.49 \pm 0.17$ & $* *$ & $0.17 \pm 0.01$ & $0.07 \pm 0.02$ & $* * *$ & $1.06 \pm 0.02$ & $1.01 \pm 0.27$ & ns \\
\hline C20:3 n3 & $0.18 \pm 0.02$ & $0.10 \pm 0.04$ & $* *$ & $0.09 \pm 0.03$ & $0.04 \pm 0.01$ & ns & $0.44 \pm 0.25$ & $0.43 \pm 0.33$ & ns \\
\hline C20:3 n6 & $0.16 \pm 0.01$ & $0.10 \pm 0.04$ & ns & nd & nd & & & & \\
\hline C20:4 n6 & $0.33 \pm 0.15$ & $0.27 \pm 0.08$ & ns & $0.11 \pm 0.01$ & $0.22 \pm 0.06$ & $* *$ & $0.59 \pm 0.21$ & $0.12 \pm 0.34$ & ns \\
\hline C20:5 n3 & nd & $0.01 \pm 0.01$ & & nd & nd & & na & & \\
\hline $\mathrm{C} 22: 0$ & $0.02 \pm 0.001$ & $0.03 \pm 0.01$ & ns & nd & $0.02 \pm 0.00$ & & na & $0.23 \pm 0.24$ & \\
\hline $\mathrm{C} 22: 5 \mathrm{n} 3$ & $0.05 \pm 0.01$ & $0.07 \pm 0.02$ & ns & nd & nd & & na & na & \\
\hline $\mathrm{C} 22: 6 \mathrm{n} 3$ & $0.01 \pm 0.002$ & nd & & nd & nd & & na & na & \\
\hline$\Sigma$ saturated & $27.07 \pm 3.08$ & $36.22 \pm 1.18$ & $* *$ & $62.81 \pm 1.45$ & $73.83 \pm 0.69$ & $* * *$ & na & na & \\
\hline$\Sigma$ unsaturated & $72.93 \pm 3.08$ & $63.78 \pm 1.18$ & $* *$ & $37.19 \pm 1.45$ & $26.17 \pm 0.69$ & $* * *$ & na & na & \\
\hline$\Sigma$ monoenoic & $49.41 \pm 0.95$ & $50.26 \pm 5.09$ & ns & $25.95 \pm 0.57$ & $21.20 \pm 0.74$ & $* * *$ & $0.23 \pm 0.02$ & $0.53 \pm 1.13$ & $*$ \\
\hline$\Sigma$ polyunsaturated & $23.52 \pm 2.56$ & $13.52 \pm 5.11$ & $* *$ & $11.24 \pm 0.89$ & $4.97 \pm 0.38$ & $* * *$ & na & na & \\
\hline$\sum \mathrm{n} 3$ & $1.34 \pm 0.11$ & $0.87 \pm 0.06$ & $* * *$ & $0.42 \pm 0.03$ & $0.29 \pm 0.02$ & $* * *$ & na & na & \\
\hline$\Sigma \mathrm{n} 6$ & $22.02 \pm 2.62$ & $12.50 \pm 5.09$ & $* *$ & $10.81 \pm 0.90$ & $4.67 \pm 0.36$ & $* * *$ & na & na & \\
\hline$\Sigma \mathrm{n} 6 / \Sigma \mathrm{n} 3$ & $16.56 \pm 3.16$ & $14.44 \pm 6.52$ & ns & $26.04 \pm 3.69$ & $16.20 \pm 0.40$ & $* *$ & na & na & \\
\hline$\sum \mathrm{n} 9$ & $46.42 \pm 0.99$ & $46.09 \pm 4.61$ & ns & $20.89 \pm 0.85$ & $14.17 \pm 0.44$ & $* * *$ & $1.04 \pm 0.08$ & $1.54 \pm 0.16$ & ns \\
\hline$\Sigma$ unsat./ $\Sigma$ sat. & $2.73 \pm 0.40$ & $1.76 \pm 0.09$ & $* *$ & $0.59 \pm 0.04$ & $0.35 \pm 0.01$ & $* * *$ & na & na & \\
\hline Average chain length & $17.57 \pm 0.04$ & $17.39 \pm 0.04$ & $* *$ & $16.64 \pm 0.04$ & $16.35 \pm 0.02$ & $* * *$ & na & na & \\
\hline Unsaturation index & $98.76 \pm 5.80$ & $78.97 \pm 5.58$ & $* *$ & $49.06 \pm 2.33$ & $31.86 \pm 0.85$ & $* * *$ & na & na & \\
\hline
\end{tabular}

HLW Hungarian Large White, nd not detected, na not available, ns not significant, ${ }^{*} P<0.05,{ }^{* *} P<0.01,{ }^{* * *} P<0.001$

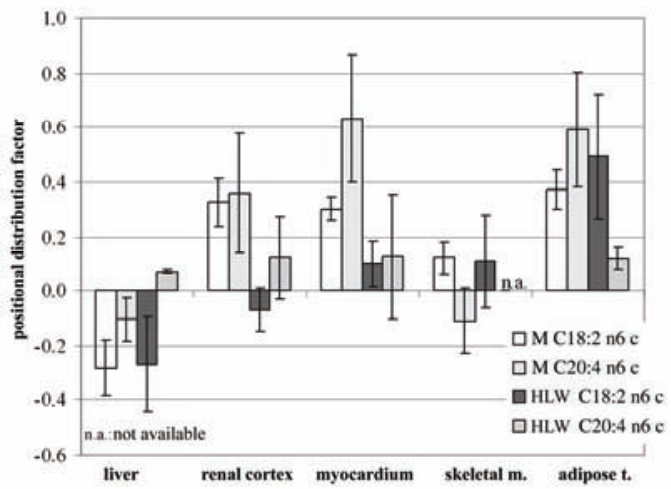

Figure 2

Positional distribution of the precursor (C18:2 n6c) and product (C20:4 n6) n6 fatty acids in the porcine tissues

Regionale Verteilung von Vorläufer und Produkt Fettsäuren 


\section{Discussion}

The two purposes of this study were to give a detailed description of the triacylglycerol molecular structure in the porcine body and to find possible differences in the TAG bioassembly in two genotypes with largely differing liponegic capacities. Present results basically support the already known rule that $\beta$-glyceride linkages of dietary fats are not retained during the porcine absorption and deposition.

Two pathways play the determinant role in the mammalian TAG synthesis: the monoacyl-glycerol and the glycerol-3-phosphate pathways. More recently, a third pathway has been outlined by LEE et al. (2001), termed the »direct acylation of glycerol«. This latter pathway has been demonstrated in myocardium, liver, kidney, brain and skeletal muscle, and its activity became prominent when the glycerol-3-phosphate pathway was suppressed. As far as the authors are aware, a wide spectral analysis taking the above aspects into consideration of porcine tissues is still lacking. Moreover, the typical Hungarian Mangalica pig has not yet been subject of detailed studies, albeit this breed gains a more and more valuable status.

Skeletal muscle neutral lipids primarily serve as oxidizable energy sources. Thus, the fatty acid distribution pattern is not only the result of synthesis, but fatty acid selective oxidation (SIDOSSIS et al. 1998) and hydrolysis (RACLOT 1997) may as well contribute to the shaping of the final composition. The results that $s n-1,3$ positions are dominantly acylated by UFAs, and the central position is occupied mostly by palmitate suggests that the synthetic course of porcine intramuscular TAG is not different from that of the adipose TAG. The turnover of IMTAG is rather active, thus the maintenance of the special TAG structure supposes a retaining condition. According to BUDOHOSKI et al. (1996) in rat redoxidative muscles that rate of TAG synthesis is assured by expressed fatty acid uptake and the high capacity TG synthesis pathway. Since the synthetic pathway of adipose tissue TAG and IMTAG is identical, pending further specific information on the enzymes involved, it might be accepted that the same acyltransferases (STOKES and TOVE 1975) are responsible for the highly similar and characteristic porcine TAG structures. In murine and human tissues the relative expression of glycerol-3-phosphate acyltransferase mRNA is high in the kidney, adipose tissue, muscle and heart, while relatively low in the liver (CAO et al. 2008). The above demonstrated specificity of porcine muscle TAG fatty acid pattern has been as well published by PETRÓN et al. (2004), in the biceps femoris of Iberian and Duroc pigs.

The genotype-associated difference, in particular the different relative occurrence of the C:18 MUFAs is highly interesting finding. Rat adipocytes have been published to equally esterify all glycerol positions with labeled oleic acid from the incubation medium (GUO et al. 1999). A near-zero (or zero) PDF value indicates a random channeling of the fatty acid to all glycerol positions, like for trans vaccenic acid by the Mangalica pigs. Thus, the higher PDF, i.e. the high relative occurrence frequency of trans vaccenic acid in the meat types pigs, as compared to the Mangalica may be handled as a new result.

The fact that the fatty acid regiodistribution of pig liver TAGs differs from that of adipose tissue has been first shown by STOKES et al. (1975). Since the TAG synthesis of liver and adipose tissue is basically similar, most probably two conditions may be hypothesized behind their different fatty acid pattern: either possible fatty acid 
selectivity of the enzymes or the prominent presence of the direct acylation pathway in one of the tissues.

The fact that in liver 2 MAGs linoleic acid was predominant suggests that the modification of the dietary TAGs, i.e. the brake of the $\beta$-glycerol linkages happens to a lesser extent »before« the liver in the pig tissues. This has been as well supported by the fatty acid distribution of the diet and stomach content TAGs, possessing very high proportions of central linoleic acid (Table 1). A further support that dietary manipulation fails to influence the fatty acid regiodistribution of the porcine liver TAGs has been provided by TISCHENDORF et al. (2002), testing the modifying effects of extra dietary CLA without any detectable effects on this fraction. In contrast, INNIS et al. (2004) found that dietary fat with randomized fatty acid distribution is a potent factor in altering the fatty acid distribution of pig hepatic TAGs.

The final or single »specifier factor" to shape the specific porcine TAG pattern is not known. According to YANG and KUKSIS (1991) ca. $70-80 \%$ of the chylomicron TAG is synthesized via the 2-monoglyceride pathway, providing little specificity in the positioning of fatty acids. Thus, there may exist two processes to shape the hepatic TAGs of pigs: the absence of the hydrolysis of the $\beta$-glycerol linkages and the dominance of the glycerol-3-phosphate pathway in the gut endothelium. This pathway is positioning longchain UFA to the central position, while the 2-monoglyceride route provides no fatty acid specificity.

Renal cortex has been less investigated for the regional distribution of its TAG fatty acids, though it has been early published that in pigs the TAG fatty acid pattern is also speciesspecific (CHRISTIE and MOORE 1970). Again, this result, and the present data suggest the extrahepatic conversion of the TAG fatty acid structure to the characteristic porcine pattern. According to LEE et al. (2001) the direct acylation of glycerol by the glycerol acyltransferase is possible in the porcine kidney microsomes. In an early report HAGEN (1971) hypothesized that the porcine renal TAG structure is the inverse of the phospholipids, assuming a common diacylglycerol precursor for the synthesis of these lipids.

Myocardial ATP production is dependent chiefly on the oxidative decarboxylation of glucose and fatty acids. The basic characteristics of the porcine myocardial TAG fatty acid pattern were largely similar to all other tissues studied, expect the liver. The ultimate reason of this phenomenon may be the direct aclytion of palmitate into position $s n-2$. It is interesting that energy deprivation also largely and selectively alters the myocardial fatty acid profile (FORD et al. 1996, SZABÓ et al. 2005), possible also contributing to the ultimate TAG fatty acid pattern. The responsible factor for the central position of palmitate has been reported to be a »specifier « interacting with acyltransferases to direct palmitate into position sn-2.

A genotype-associated difference was found by the presence of DPA merely in the 2MAGs of Mangalica pigs. Obviously, the source of DPA in tissue lipids was not the diet, thus endogenous desaturation and elongation of a-linolenic acid (ALA) may be the route to form DPA. Though there is a lack of direct information on the ability of different swine breeds to transform fatty acids, a five-fold higher precursor (i.e. ALA) supply has been proven for DPA in the Mangalica diet. This dietary provision is potent in increasing the 
TAG DPA levels (NELSON and ACKMAN 1988), but it still remains an unresolved issue, how this longchain PUFA was directed to the $s n-2$ position, even if in a rather low proportion.

Porcine adipose tissue is the mostly studied tissue from the aspect TAG fatty acid profile (NÜRNBERG et al. 1994, BIEDERMANN et al. 2000, GLODEK et al. 2004). In the present study the centrally abundant palmitic acid generally agrees with earlier published results (MATTSON et al. 1964). However, the exclusive presence of palmitate in the 2MAGs of Mangalica pigs seems to be a unique trait. In an earlier approach (SZABÓ et al. 2007) extensively kept meat type pig and also wild boar adipose tissue was analyzed, with ca. $10 \%$ palmitate in the 1,3 positions, in both genotypes. MATTSON et al. (1964) detected $86-92 \%$ of total palmitate in the 2 MAGs of domestice pigs, while $81-88 \%$ was found for the wild boar. Similarly, KING et al. (2004) found serious amounts of palmitic acid in the $s n-1,3$ positions in the subcutaneous adipose tissue of CLA fed pigs. Palmitic acid is the final product of the mammalian de novo fatty acid synthesis, but is as well abundant in feed lipids (Table 1). Thus, the exact source of the acids found in the tissue sample is not known. Since the proportion of palmitate in the Mangalica 2MAG fatty acid profile was markedly lower than in the meat type pig, it may as well be assumed that the channeling of palmitate might reach a critical level over which the 1,3 positions of glycerol are also acylated. However, this seems to contrast with the markedly higher palmitate proportion (86-92\%) in the 2MAGs, as reported by MATTSON et al. (1964).

In addition, KING et al. (2004) published that dietary conjugated linolenic acid (CLA) is potent in changing the fatty acid distribution pattern, in contrast to its neglectable effects on liver TAGs. This was especially true for palmitate and stearate, tending towards positions 1 and 3, as a result of added dietary CLA. In this study the dietary CLA levels were identical for both genotypes, thus this effect can not be taken into account in the determination of the palmitate position.

Pending further results in this field, Mangalica seems to represent a special trait, directing the entire palmitate moiety into the central position of its TAGs. An ultimate explaining of the finding may be that hormone sensitive lipase (HSL) shows relative, although not absolute positional specificity, preferring fatty acids at $s n-1,3$. Moreover, from within the fatty acids of identical saturation level (e.g. SFAs) the shorter chains are more quickly hydrolyzed.

As two further organs analyzed, the spleens and the lungs were as well studied. The main characteristic, the central recruitment of palmitate was found in both tissues, without any differences between genotypes. As far as the authors are aware, swine pulmonary and spleen TAGs have not yet been investigated from this aspect, and showed the distinctive porcine TAG pattern.

In conclusion, the direct analysis results of the 2MAGs in six extrahepatic organs and in the liver provided evidence that the building-up of the characteristic porcine TAG structure is located at a post-hepatic site, being true for most of the organs of the pig body. Mangalica has been found to provide a highly similar TAG fatty acid bioassembly to the so-far analyzed breeds, with minor differences, primarily in the adipose tissue. 


\section{Acknowledgements}

The support of the Bolyai Research Grant (BO/00108/2007) to A. Szabó. by the Hungarian Academy of Sciences is gratefully acknowledged.

\section{References}

Biedermann G, Jatsch C, Peschke W, Lindner JP, Wittmann W (2000) Fattening and carcass Performance and meat and fat quality of Pietrain pigs of different MHS Genotype and sex. II. Fatty acid pattern of the adipose tissues back fat, leaf fat and intramuscular fat and of the total- and phospholipids of the m. long. dorsi. Arch Tierz 43, 165-78 [in German]

Brockerhoff H, Ackmann RG (1967) Positional distribution of isomers of monoenoic fatty acids in animal glycerolipids. J Lipid Res 8, 661-66

Budohoski LJ, Gorski K, Nazar H, Kaciuba-Uscilko J, Terjung RL (1996) Triacylglycerol synthesis in the different skeletal muscle fiber sections of the rat. Am J Physiol 271, 574-81

Cao J, Shan D, Revett T, Li D, Wu L, Liu W, Tobin JF, Gimeno RE (2008) Molecular identification of a novel mammalian brain isoform of acyl-CoA:lysophospholipid acyltransferase with prominent ethanolamine lysophospholipid acylating activity, LPEAT2. J Biol Chem 283, 19049-57

Christie WW (1982) A simple procedure for rapid transmethylation of glycerolipids and cholesteryl esters. J Lipid Res 23, 1072-5

Christie WW, Moore JH (1970) A comparison of the structures of triglycerides from various pig tissues. Biochim Biophys Acta 210, 46-56

Ender K, Nurnberg K, Wegner J, Seregi J (2002) Laboratory investigations of Mangalitza meat and fat. Fleischwirtschaft 82, 125-8

Folch, J Mangalica, Lees Mangalica, Sloane-Stanley GH (1957) A simple method for the isolation and purification of total lipids from animal tissues. J Biol Chem 226, 495-509

Ford DA, Han X, Horner CC, Gross RW (1996) Accumulation of unsaturated acylcarnitine molecular species during acute myocardial ischemia: metabolic compartmentalization of products of fatty acyl chain elongation in the acylcarnitine pool. Biochemistry 35, 7903-9

Glodek P, Kratz R, Schulz E, Flachowski G (2004) Effect of sire breeds in commercial pig crosses on growth, carcass composition, meat and fat quality. Arch Tierz 47, 59-74 [in German]

Guo W, Choi JK, Kirkland JL, Corkey BE, Hamilton JA (1999) Incorporation of [1-13C]oleate into cellular triglycerides in differentiating 3T3L1 cells. Lipids 34, 825-31

Hagen PO (1971) Structural comparison between triglycerides and phospholipids from pig kidney. Lipids 6, 935-41

Hilditch TP Stainsby WJ (1935) The body fats of the pig: Progressive hydrogenation as an aid in the study of glyceride structure. Biochem J 29, 90-9

Innis SM, Dyer R, Quinlan P, Diersen-Schade D (1995) Palmitic acid is absorbed as sn-2 monopalmitin from milk and formula with rearranged triacylglycerols and results in increased plasma triglyceride sn-2 and cholesteryl ester palmitate in piglets. J Nutr 125, 73-81

Innis SM, Dyer R, Quinlan P, Diersen-Schade D (1996) Dietary triacylglycerol structure and saturated fat alter plasma and tissue fatty acids in piglets. Lipids 31, 497-505

King DA, Behrends JM, Jenschke BE, Rhoades RD, Smith SB (2004) Positional distribution of fatty acids in triacylglycerols from subcutaneous adipose tissue of pigs fed diets enriched with conjugated linoleic acid, corn oil, or beef tallow. Meat Science 67, 675-81

Lee DP, Deonarine AS, Kienetz Mangalica, Zhu Q, Skrzypczak Mangalica, Chan Mangalica, Choy PC (2001) A novel pathway for lipid biosynthesis: the direct acylation of glycerol. J Lipid Res 42, 1979-86

Litchfield C (1972) Analysis of triglycerides. Academic Press, New York, USA.

Mattson FH, Volpenhein RA, Lutton ES (1964) The distribution of fatty acids in the triglycerides of the Artiodactyla (even-toed animals). J Lipid Res 5, 363-5

Nelson GJ, Ackman R (1988) Absorption and transport of fat in mammals with emphasis on $n-3$ polyunsaturated fatty acids. Lipids $23,1005-14$

Nürnberg, K, Kulm G, Ender K, Nürnberg G (1994) Effect of porcine somatotropin on carcass quality and adipose tissue composition in genetically different pigs. Arch Tierz 37, 265-78 [in German]

Petrón MJ, Muriel E, Timóna ML, Martína L, Antequera T (2004) Fatty acids and triacylglycerols profiles from different types of Iberian dry-cured hams. Meat Sci 68, 71-7 
Raclot T (1997) Selective mobilization of fatty acids from white fat cells: evidence for a relationship to the polarity of triacylglycerols. Biochem J 322, 483-9

Sidossis LS, Wolfe RR, Coggan R (1998) Regulation of fatty acid oxidation in untrained vs. trained man during exercise. Am J Physiol 274, 510-5

SPSS 10 (1999). SPSS Inc. Chicago, IL, USA

Stokes GB, Tove SB (1975) Evidence for a factor in pig adipose tissue controlling the specificity of the acyltransferase(s) of triacylglycerol synthesis. J Biol Chem 25, 6315-9

Stokes GB, Poteat LW, Tove SB (1975) Distribution of fatty acids incorporated into triacylglycerols by microsome/cytosol preparations from adipose tissue. Biochim Biophys Acta 380, 245-56

Szabó A, Fébel H, Sugár L, Romvári R (2007) Fatty acid regiodistribution analysis of divergent animal triacylglycerol samples - a possible approach for species differentiation. J Food Lipids 14, 62-77

Szabó A, Fébel H, Mézes M, Horn P, Balogh K, Romvári R (2005) Differential utilization of hepatic and myocardial fatty acids during forced molt of laying hens. Poultry Sci 84, 106-12

Tischendorf F, Möckel P, Schöne F, Plonné Mangalica, Jahreis G (2002) Effect of dietary conjugated linoleic acids on the distribution of fatty acids in serum lipoprotein fractions and different tissues of growing pigs. J Anim Physiol Anim Nutr 86, 313-25

Yang LY, Kuksis A (1991) Apparent convergence (at 2-monoacylglycerol level) of phosphatidic acid and 2-monoacylglycerol pathways of synthesis of chylomicron triacylglycerols. J Lipid Res 32, 1173-86

Received 4 May 2009, accepted 6 January 2010.

Corresponding author:

ANDRÁS SZABÓ

email: szan1125@freemail.hu

Laboratory of Animal Product Qualification, Faculty of Animal Science, Kaposvár University, 7400,

Kaposvár, Guba S. u. 40, Hungary 